\title{
The effects of electrically exploding gold bridgewires into inert and explosive powder beds
}

\author{
P. J. $\operatorname{Rae}^{1}$ (1) $\cdot$ R. C. Rettinger ${ }^{1}$
}

Received: 26 April 2021 / Revised: 21 August 2021 / Accepted: 18 September 2021 / Published online: 23 October 2021 The Author(s) 2021

\begin{abstract}
The particle velocity created in beds of both low-density inert sugar and explosive PETN as a function of distance from an exploding bridgewire was measured using optical velocimetry and a silvered PMMA window. As expected, more violent bridge-bursts (from a greater-stored-energy capacitive discharge unit) resulted in greater particle velocities and a better supported compaction wave in sugar. In all cases, ramp waves, not shocks, were observed in the inert sugar. Large window velocities were observed for very powerful bursts (up to $270 \mathrm{~m} / \mathrm{s}$ ), but bursts required for stochastic detonator operation conditions resulted in sugar/PMMA window velocities of only $8-10 \mathrm{~m} / \mathrm{s} 0.85 \mathrm{~mm}$ from the bridge location. In contrast, after a distance of only $0.65 \mathrm{~mm}$, a building shock wave was observed in PETN under both threshold and reliable firing conditions. Subsequently a hot-spot-driven shock-to-detonation (SDT) process was observed prior to full detonation. The measured buildup process accounts for $\approx 66 \%$ of the so-called excess transit time (ETT) between the observed and theoretical total function time for the particular exploding-bridge-wire (EBW) detonator studied. The remainder must occur in the powerful output pellet region. In contrast to a common understanding, the ETT is found to be a weak function of the discharge energy. Thus, the operation of the detonator after a bridge-burst energy-to-powder reaction transition process is found to be hot-spot-driven SDT in both the low- and high-density pellets.
\end{abstract}

Keywords Detonator · Bridgewire · Shock compaction · PETN $\cdot$ Powder

\section{Introduction}

The exploding bridgewire (EBW) detonator was invented in Los Alamos toward the end of the Second World War [1]. The aim was to create a safe detonator with highly repeatable functioning time (less than $1 \mu \mathrm{s}$ of jitter) coupled to a powerful explosive booster output.

This was achieved by discharging a high-voltage capacitor across a short, very fine gold wire to create a shock wave, gold plasma, and arc next to a pellet of low-density $(\approx 50 \%$ of theoretical maximum density, TMD) PETN (2,2-bis[(nitrooxy)methyl]propane-1,3-diyl dinitrate). The exploding of the wire, also called bridge-burst, was discovered to reproducibly detonate the low-density PETN (the initial pressing or IP) by some mechanism, and by cou-

Communicated by P. Hazell.

$\triangle$ P. J. Rae prae@lanl.gov

1 M-6, Los Alamos National Laboratory, PO Box 1663, MS-P917, Los Alamos, NM 87545, USA pling this low-pressure detonation output to a higher-density booster pellet (the output pellet or OP), a safe, reliable, highly reproducible detonator was made containing only secondary explosives. Subsequently it was discovered that some other explosives such as RDX, HMX, and CL-20 can be used instead of PETN although the electrical energy required for detonation generally increases, while others such as HNS will not work ${ }^{1}$. This paper will concentrate on PETN since it is the most commonly used although future research may study other explosives.

Despite extensive use for 70 years, there is still some uncertainty about exactly how EBW detonators actually function. It is probable that several effects contribute to produce a prompt volumetric thermal explosion in the lowdensity powder bed that transitions into a detonation [2]. Proposed energy sources for the thermal explosion include shock from the bridgewire explosion, $P \mathrm{~d} V$ work from compaction of the porous bed, thermal energy from the arc,

${ }^{1}$ RDX: 1,3,5-trinitroperhydro-1,3,5-triazine, HMX: 1,3,5,7-tetranitro1,3,5,7-tetrazocane, CL-20: hexanitrohexaazaisowurtzitane, HNS: 1,1((e)-ethane-1,2-diyl)bis(2,4,6-trinitrobenzene). 
and photochemical/direct-energy absorption in the explosive of the UV light produced by the arc. Recent research has demonstrated that synergistic interaction between shock compaction and photochemical effects is required to function the detonator in the usual manner [3].

A closely related detonator type is the arc detonator (AD) $[4,5]$. This is essentially identical to an EBW detonator except that there is no bridgewire and the electrical arc jumps across the gap between two terminals. ADs have been shown to function at lower energies than similar EBWs, but a special capacitor discharge unit (CDU) is required with a very low inductance, fast turn-on, high-voltage switch. For this reason, among others, they are not in common usage, but it does demonstrate that the energy from an arc alone is sufficient to produce detonation in a PETN IP.

As a result, it has been proposed that the purpose of the bridge is to act as a fuse that allows the current from the CDU to ramp to a sufficient magnitude such that when the bridge begins to burst and the resistance climbs rapidly, the resulting inductive voltage kick forms an arc and burst with a power great enough that it results in a reasonably prompt detonation in the powder $[4,6]$. The bridge also provides greater safety from accidental detonation caused by the inadvertent application of high-voltage electrical stimuli (e.g., carpet or furniture shocks) than the equivalent $\mathrm{AD}$.

Bridges made from gold are the most common, but other materials such as aluminum, copper, silver, iron, platinum, and tungsten have all been used successfully $[7,8]$. The most efficient EBW wire materials have low boiling points, low heats of vaporization, and a high increase in resistance at burst [9].

There is a minimum electrical energy at wire burst required to stochastically function an EBW detonator [10]. As this burst energy is systematically raised above this threshold, an increasing fraction will detonate, but initially with relatively poor timing reproducibility. This is termed the soft-fire region of performance. The threshold value (usually expressed as a voltage rather than an energy) is defined as the level required to function $50 \%$ of the detonators tested. As the energy is raised further, an asymptotic common minimum functioning time (electrical burst until detonation breakout at the end of the detonator) will be found. This is termed the hard-fire condition. It is therefore found that increasing the energy still further will not significantly reduce the function time. Since the energy stored on a capacitor is $\frac{1}{2} C V^{2}$, it is usually most convenient to increase the energy at burst by increasing the initial charge voltage, although both the system inductance and capacitance can also be altered to optimize a specific CDU and detonator combination.

The purpose of this research was to examine the interaction of the exploding wire shock and arc with both an inert and an explosive powder bed. The particle velocity imposed on a PMMA (poly-methyl methacrylate) window in contact with the powder was recorded using photonic Doppler velocimetry (PDV) $[11,12]$ to measure the motion imparted to the porous bed by the wire burst, the arc process, and any reaction from the explosive. In this way, the relative energy for different firing conditions, the presence or absence of a wire, firing cable inductance, and explosive buildup to detonation could be quantified. One useful technique to study this problem is to reduce the powder thickness from its nominal detonator dimension to just a thin layer surrounding the bridge where a measurement is then made. This technique is termed cutting the detonator back, or simply cut-back tests.

Additionally, this paper reassesses some of the previously stated facts about EBW detonator functioning as a result of both new information presented here and the recent availability of interior density radiographic, rather than solely externally obtained optical images. The result is a significant change in the explanation for the mode of operation of EBW detonators.

\section{Experimental setup}

A standard commercial detonator header (RP-1, Teledyne RISI, www.teledynerisi.com) with an empty brass sleeve had thin layers of $0.88 \mathrm{~g} / \mathrm{cc}$ either confectioners sugar or high specific surface area PETN pressed against the bridgewire using a precision die-set and press. Confectioners sugar has been previously shown to result in the same electrical signals at burst as PETN, but being a largely inert organic crystal as opposed to an organic energetic one, the compaction in the powder bed is a function of only the drive from bridge-burst [13]. In fact, in contrast to the highly exothermic process of reaction in PETN, the breakdown of sugar will be endothermic. The PETN powder used was also supplied by RISI and was the high surface area powder for regular RP-1s.

The gold bridgewire is $38 \mu \mathrm{m}$ in diameter ( 0.0015 inches) by $1.02 \mathrm{~mm}$ long ( 0.040 inches). A 4-mm-thick PMMA window aluminized with a $1 \mu \mathrm{m}$ layer on one side and a $1550-\mathrm{nm}$

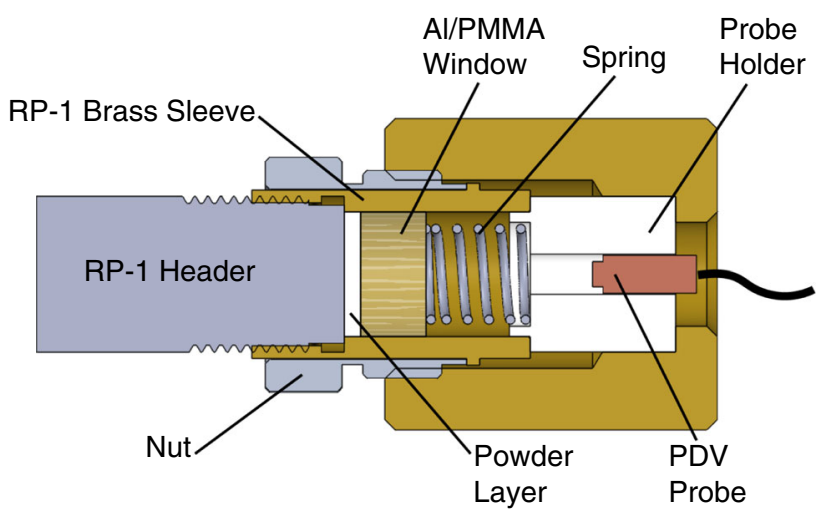

Fig. 1 The cross section of the experimental arrangement 
anti-reflection coating (Knight Optical, www.knightoptical. com) on the free surface was held in contact with the sugar using light spring pressure, see Fig. 1. The thickness of the powder was varied between 0.5 and $6.15 \mathrm{~mm}$ by the use of precision pressing shims. The mass of the powder was adjusted to maintain a consistent density. It was discovered that a layer less than $0.5 \mathrm{~mm}$ could not be reliably pressed.

The crystal density of sugar is $1.59 \mathrm{~g} / \mathrm{cc}$ compared with $1.77 \mathrm{~g} / \mathrm{cc}$ for PETN. The powdered sugar had a particle size of approximately $10 \mu \mathrm{m}$. A typical IP density of PETN is $0.88 \mathrm{~g} / \mathrm{cc}(49.7 \%$ of TMD), and this density was matched with the inert sugar pressings.

The CDU used for the inert sugar tests had a $5 \mu \mathrm{F}$ capacitor capable of being charged between 0.6 and $3.9 \mathrm{kV}$ and triggered by an optical signal. A short $(0.3 \mathrm{~m})$ cable from the CDU to the test header was used for the majority of the tests to minimize inductance and current rise time. For one test, a longer $13 \mathrm{~m}$ cable was used to be more representative of a real firing site configuration. Firing voltages of 3.5, 1.8, 1.2 , and $0.9 \mathrm{kV}$ were used. A charge voltage of $3.5 \mathrm{kV}$ and the short cable hugely over drove the header compared with normal firing conditions. Charge voltages for this oversized CDU of $1.8-0.9 \mathrm{kV}$ are much more representative of typical firing conditions actually used.

A different on/off CDU was used for the PETN and some sugar powder tests. This CDU also used a $5 \mu \mathrm{F}$ capacitor in series with a fast SiC MOSFET switch. The switch was a Cree model CAS120M12BM2 rated at $1.2 \mathrm{kV}$ with a CGD15HB62 driver circuit (wolfspeed.com). The maximum transient current rating is more than $480 \mathrm{~A}$, and it has a rated turn-on/turn-off time of $70 \mathrm{~ns}$ when driving a very low inductance circuit. To avoid over-voltaging the switch at burst time, the CDU was only charged to $600 \mathrm{~V}$ and a flywheel diode was placed across the switch in case the output was under-damped. This CDU was chosen because the conduction time of the current through the bridge could be accurately controlled allowing the effects of the large postburst energy available on the buildup of reaction in PETN to be assessed. Using this large-capacitance, low inductance CDU charged to a modest $600 \mathrm{~V}$, no more than 1us of total programmed conduction time was needed to hard-fire the bridgewire [10].

With both CDUs, the output current was measured with an integral $20 \mathrm{MHz}$ bandwidth Pearson 5046 device (pearsonelectronics.com) and the voltage across the copper leadwires by a $50 \mathrm{MHz}$ bandwidth CalTest CT4079-NA floating differential high-voltage probe using an attenuation factor of 4000 or 400 depending on the charge voltage. Due to the inductance of these copper leadwires, the voltage measured at this location differs from that across the gold bridgewire, but practically it is extremely difficult to make an accurate measurement inside the header. Instead we measured the inductance of the detonator lead loop $(L)$, and the measured voltage was corrected to a bridge voltage using the voltage across an inductor $V=-L \mathrm{~d} i / \mathrm{d} t$. The signals were digitized on either a Tektronix DSO4104B oscilloscope at $5 \mathrm{GS} / \mathrm{s}$ or a Tektronix MSO58 digitizer at $1.25 \mathrm{GS} / \mathrm{s}$.

The velocity of the powder/PMMA window was measured using a heterodyne $1550 \mathrm{~nm}$ up-shifted PDV system with an initial fringe frequency of $\approx 1 \mathrm{GHz}[12,14]$. An AC Photonics (www.acphotonics.com) fiber-pigtailed collimated probe ( $0.4 \mathrm{~mm}$ beam diameter) was used to measure the particle velocity in the center of the $7.62 \mathrm{~mm}$ diameter by 4 -mm-thick window. The window size is adequate to prevent side releases or rear free-surface motion for at least $0.5 \mu$ s ensuring that the velocity measured is imparted solely by the powder bed pressure for that duration. The bridge-burst is highly divergent, and therefore, the measured velocity of the window interface at the center will be an integrated function of this complex input. It is not known what thickness of powder is required to spread the divergent compaction wave into a temporary steady-state in the powder, but it is probable that the shock releases from the rear rather than the side resulting in only a brief transitory 1D steady-state condition. The refractive index fringe velocity correction for PMMA is 1.0 [15], and so no correction was performed on the data analyzed using a short-time fast Fourier transform (sFFT) method [14].

We wished to explore the output from an arc and so a header that had previously been tested was used to make an arc detonator (i.e., the gold wire was no longer present). A CDU voltage of $3.5 \mathrm{kV}$ is not sufficient to reliably operate in arc mode with a 1.02-mm-long gap between electrodes. Therefore, a very fine graphite trace (using an HB pencil) was added between the electrodes before the sugar was pressed to enhance the ability of the arc to form. The vaporization temperature of graphite $(>4000 \mathrm{~K}$ ) is much higher than gold $(\approx 2900 \mathrm{~K})$ at STP, and the mass of graphite added was significantly lower than in a gold wire. It is therefore assumed that the effect of any graphite electrical explosion was minimal compared with the quickly formed arc.

\section{Results and discussion}

\subsection{Inert powder studies}

Figure 2 presents the particle velocity versus time traces for all of the inert bursts. For clarity, individual groups of traces will be separated out in later figures and discussed. It is clear from this full compilation that in all cases the PMMA window was acted on by a ramp wave, not a sharp shock. The steepness of the ramp does vary depending on the conditions. It is also clear that the imposed velocities are significant $(70-270 \mathrm{~m} / \mathrm{s})$ suggesting relatively high pressures are acting on the powder bed. 


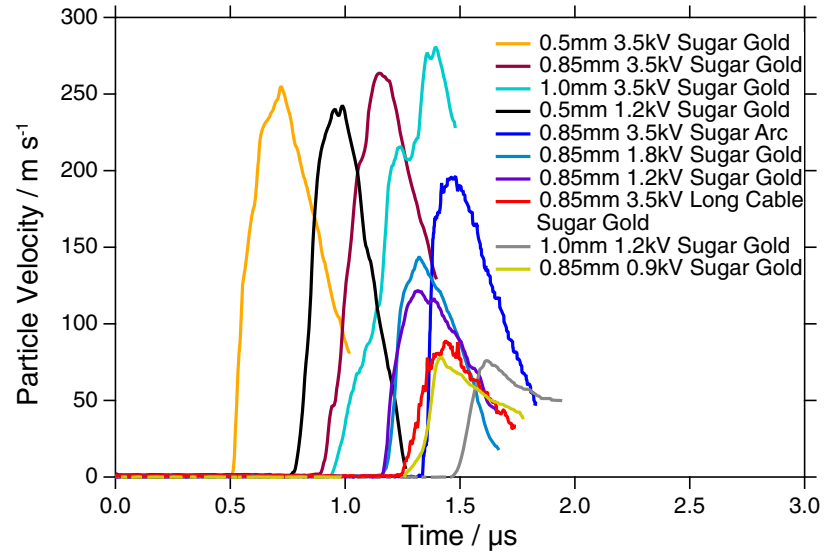

Fig. 2 Particle velocity versus time for all inert bursts. For improved clarity, individual groups of data will be presented later

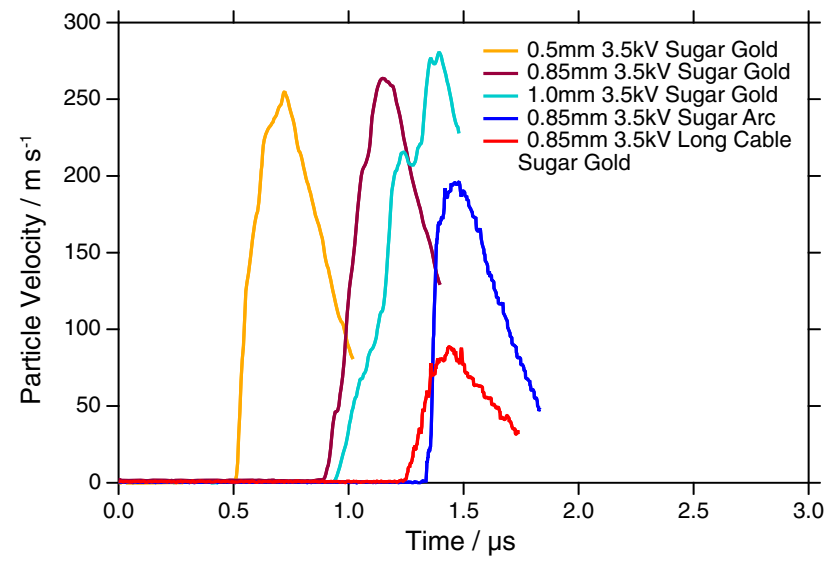

Fig. 3 Particle velocity versus time for all inert bursts at $3.5 \mathrm{kV}$

Figure 3 presents five particle velocity traces when the CDU was charged to $3.5 \mathrm{kV}$. This is an extremely energetic firing condition relative to usual practice. It is apparent that the powder thickness affects the transit time for the short cable gold-bridged headers as intuition would suggest; however, the magnitude of the peak-wave velocity is relatively unaffected suggesting that the compaction wave is largely supported by post-burst CDU electrical energy under these extreme burst conditions. It is also apparent that firing the header in arc mode delayed the arrival of the ramp-wave by approximately $450 \mathrm{~ns}$ but lowered its magnitude at $0.85 \mathrm{~mm}$ by only $\approx 25 \%$.

Example current and voltage traces for the $3.5 \mathrm{kV}$ bursts are shown in Fig. 4 together with a $1.2 \mathrm{kV}$ burst for comparison. All of the following curves have been time shifted so that bridge-burst (or voltage maximum in the arc mode case) occurs at $0 \mu \mathrm{s}$. The PDV data have also been time shifted so they are relative to burst time. Power and energy traces for $3.5 \mathrm{kV}$ bursts are shown in Figs. 5 and 6 . The current and voltage traces for a gold wire burst and an arc are very different. In the case of the arc, the voltage builds to a high initial value
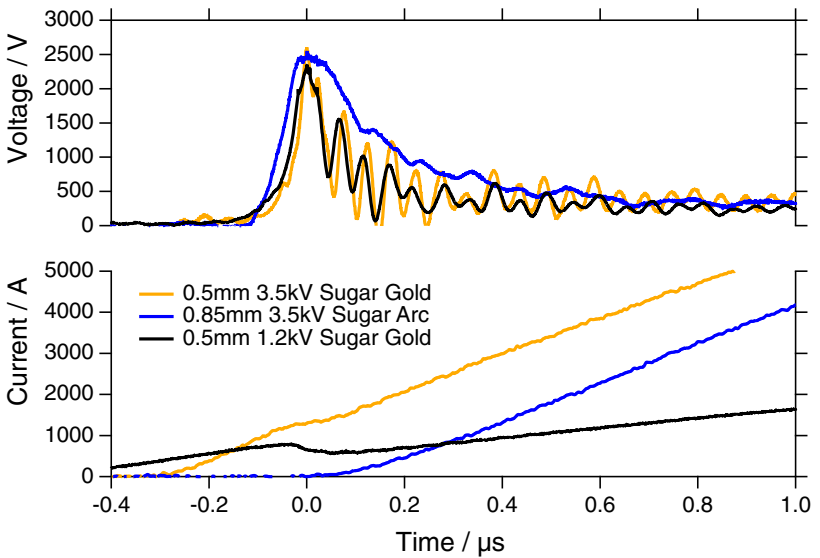

Fig. 4 Current and voltage traces for two headers at $3.5 \mathrm{kV}$ and one at $1.2 \mathrm{kV}$ in sugar. All time zeros have been corrected to bridge-burst, and the voltages have been corrected for the leadwire inductance effect

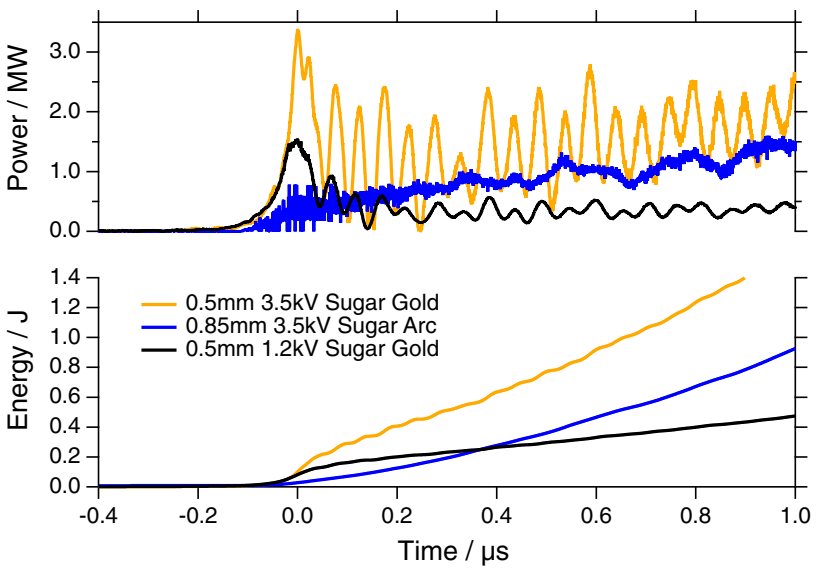

Fig. 5 Power and energy traces for two inert headers at $3.5 \mathrm{kV}$. One had a gold wire, and one had a graphite bridge

of $2400 \mathrm{~V}$ but significant current flow only begins $100 \mathrm{~ns}$ later when an arc across the terminals has formed. In the case of a gold wire, the current and voltage build together until a clear bridge-burst when the voltage spikes due to inductance effects as the current magnitude briefly plateaus.

As might be expected from an arc, a burst indication is not observed. The more reasonable firing condition of $1.2 \mathrm{kV}$ included in Fig. 4 shows a lower magnitude version of the $3.5 \mathrm{kV}$ gold wire burst.

The power and energy traces for a gold wire and an arc are also significantly different, see Fig. 5. In the gold wire, the peak power at burst is almost $3.5 \mathrm{MW}$ while the arc power shows a slowly rising value. The energy trace for the gold wire shows a rapid increase at burst while nothing similar is observed for the arc at any time.

As might be anticipated, the longer cable between the CDU and the header had a significant effect. The $3.5 \mathrm{kV}$ gold wire power trace in Fig. 6 shows oscillations as the cable rings up due to the finite velocity of current propagation in the 


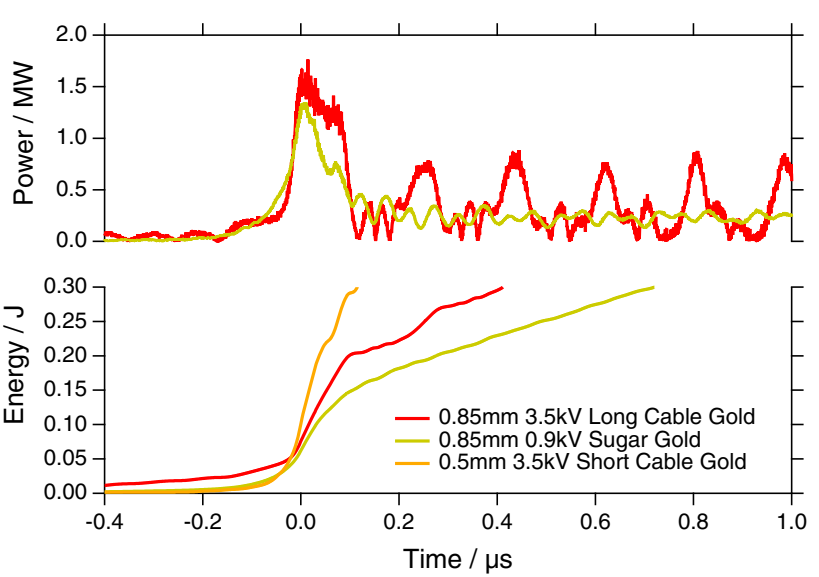

Fig. 6 Example power and energy traces for two inert headers with $0.85 \mathrm{~mm}$ of sugar exploded at different voltages and cable lengths. For comparison, the short cable $3.5 \mathrm{kV}$ burst energy shown in Fig. 5 is also shown

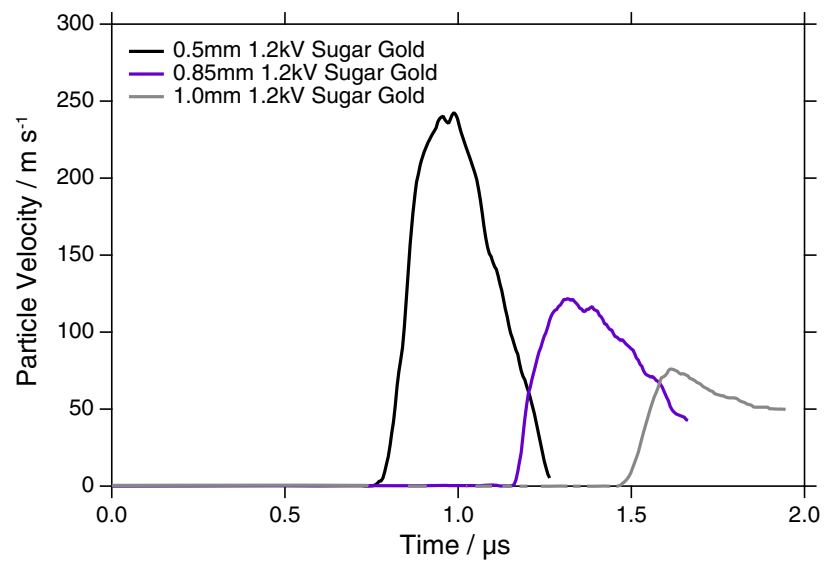

Fig. 7 Particle velocity versus time for all inert bursts at $1.2 \mathrm{kV}$

cable prior to burst. The power at burst is less than half that in the short cable, but still $\approx 1.6 \mathrm{MW}$. For comparison, Fig. 6 includes the much more modest burst generated by a $0.9 \mathrm{kV}$ CDU charge (only $6.6 \%$ of the $3.5 \mathrm{kV}$ energy) into the $0.3 \mathrm{~m}$ cable. The power at burst was also over 1.2 MW, although the energy deposited after burst is lower. Also copied for reference from Fig. 5 is the significantly greater rate and magnitude of the energy deposited at burst for the shorter cable $3.5 \mathrm{kV}$ variant.

Figure 7 presents the particle velocity data for a reasonable CDU charge of $1.2 \mathrm{kV}$ (an example voltage and current trace is shown in Fig. 4). As with the $3.5 \mathrm{kV}$ data, the transit time scales with sugar thickness; however, in this case the magnitude of the velocity drops significantly with distance suggesting the input is not as well supported by the lower post-burst energy available.

A comparison of all the traces taken with a $0.85-\mathrm{mm}$ powder bed is shown in Fig. 8. Clearly the magnitude of the velocity diminishes with lower CDU charge voltage.

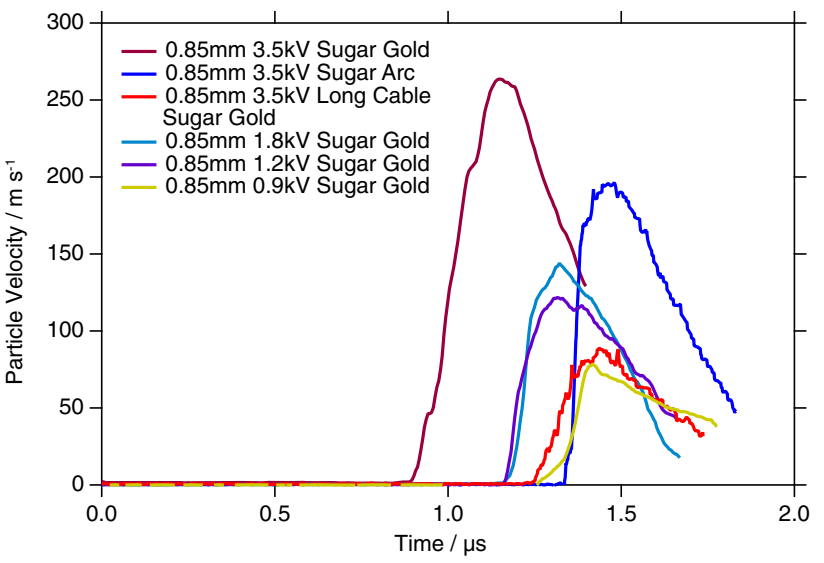

Fig. 8 Particle velocity versus time comparison for all inert bursts at $0.85 \mathrm{~mm}$

The PMMA particle velocity from the long cable burst at $3.5 \mathrm{kV}$ appears very low compared with what one might expect from the energy stored in the CDU. Indeed, the velocity profile is very similar to that generated by a $0.9 \mathrm{kV}$ CDU charge and the short cable. A partial explanation may be found in Fig. 6 where it is observed that the extra inductance of the longer cable significantly delays the delivery of the extra stored energy after burst compared with the short cable CDU charged to $0.9 \mathrm{kV}$. This implies that the CDU energy is released too slowly in the case of the longer cable to fully support the attenuating compaction wave and keep the velocity constant. However, it should be understood that a $5 \mu \mathrm{F}$ CDU charged to $3.5 \mathrm{kV}$ with a $13-\mathrm{m}$ firing cable will still result in a very hard-fire situation in a real RP-1 detonator. Thus, a PMMA particle velocity of approximately $75 \mathrm{~m} / \mathrm{s}$ after transiting $0.85 \mathrm{~mm}$ of sugar is indicative of a burst that would have resulted in a very hard-fire in a real detonator. The minimum particle velocity at an inert powder/PMMA interface that would have resulted in a detonation if instead PETN had been present is discussed in more detail later.

Previous research on cut-back real detonators coupled with velocimetry (tests similar to those presented here, but with explosive powders) has shown the presence of ramp waves of growing strength further from the bridge prior to the onset of steady detonation at $1-3 \mathrm{~mm}[16,17]$. It was assumed that this ramp wave resulted both from the behavior of low-density powder from unsupported shocks and from building reaction in the explosive behind the leading part of the ramp. That is, a compaction wave was shocking up from exothermic chemistry and eventually transitioned to a detonation. Indeed, these sugar-based experiments will contribute to understanding how much of the initial stage powder particle velocity in real detonators comes from the bridgeburst event (apparently relatively little) and how much comes from the exothermic, explosive action of the PETN chemical decomposition (the majority). 
Table 1 Approximate sugar compaction shock wave velocities at three CDU charge voltages

\begin{tabular}{lll}
\hline Charge voltage/kV & Distance span/mm & Shock velocity/m/s \\
\hline 3.5 & $0-0.5$ & 990 \\
1.2 & $0-0.5$ & 640 \\
0.6 & $0-0.5$ & 590 \\
0.6 & $0.5-0.85$ & 350 \\
3.5 & $0.5-1.0$ & 775 \\
1.2 & $0.5-1.0$ & 710 \\
0.6 & $0.85-1.0$ & 390 \\
\hline
\end{tabular}

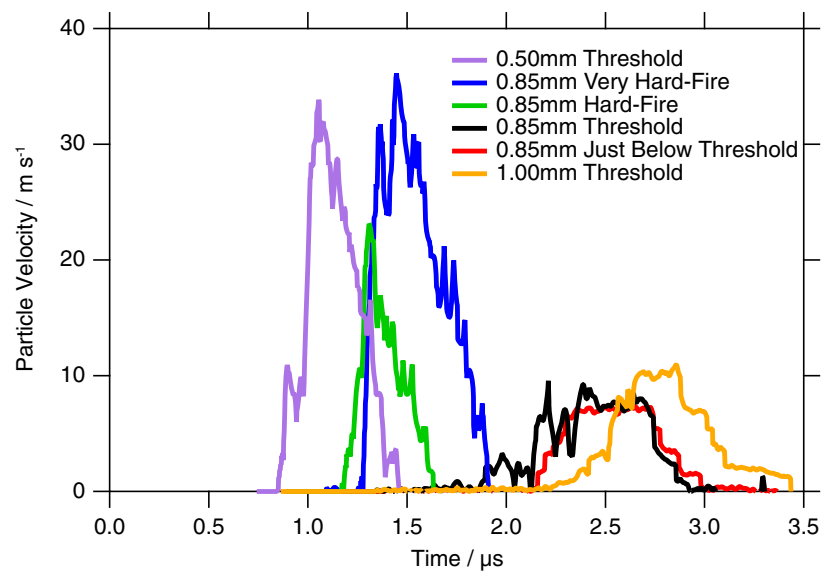

Fig. 9 Particle velocity versus time for bursts into sugar when the conduction time was varied to span the range from just below threshold to very hard-fire in an RP-1 detonator. The powder thickness was also varied

It is therefore interesting to consider what effect the initially somewhat inert compaction wave resulting from bridge-burst will have on the functioning of a real detonator. Approximate sugar compaction shock wave velocities can be estimated from the time to peak particle velocity in the PMMA window as a function of distance from the bridge using the measured electrical bridge-burst offset times. Table 1 shows these results. It is noted that all the values are under $1 \mathrm{~mm} / \mu \mathrm{s}$ and that the majority are between $0.6-0.8 \mathrm{~mm} / \mu \mathrm{s}$.

It is well known that the function time of well-designed EBW detonators in the hard-fire voltage region is very reproducible. This implies that the physical processes driving thermal explosion are not significantly accelerated by the addition of extra energy above a certain minimum threshold. Observations in the lower voltage soft-fire region have demonstrated that detonation breakout occurs in a random location, often off the bridgewire axis compared with a consistent location directly above the bridgewire in the case of hard-fires [18].

Previous research has identified the conduction time required for the $600 \mathrm{~V}$ on/off $\mathrm{CDU}$ to span the range of
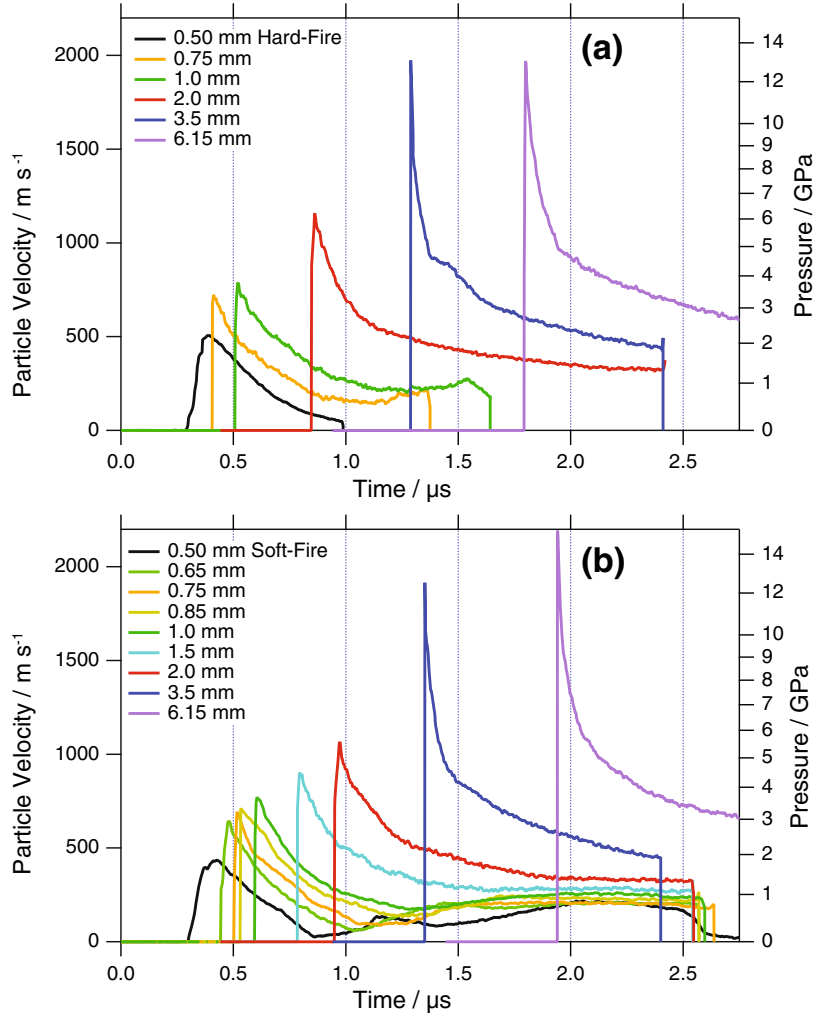

Fig. 10 Particle velocity versus time for bursts into $0.88 \mathrm{~g} / \mathrm{cc}$ PETN as a function of powder thickness: $\mathbf{a}$ a hard-fire and $\mathbf{b}$ a very soft fire. The right axis shows the slightly nonlinear pressure imparted by the corresponding particle velocity in PMMA

just below threshold, threshold, and hard-fire conditions for an RP-1 detonator [10]. Figure 9 shows the effect of these representative conduction times as a function of powder thicknesses for inert sugar pressings also of $0.88 \mathrm{~g} / \mathrm{cc}$ density. There is a significant change in particle velocity impedance matched into the PMMA window between hard-fire and just below threshold for a sugar layer thickness of $0.85 \mathrm{~mm}$. Further, it will be observed that there is not a marked difference between the velocity through $0.85 \mathrm{~mm}$ of sugar for a threshold and just below threshold burst. Consulting Table 1 for the $600 \mathrm{~V}$ CDU reveals the large reduction in shock velocity versus distance for the threshold burst case suggesting at this low burst energy the compaction wave is not well supported.

\subsection{Explosive powder studies}

Using the $600 \mathrm{~V}$ on/off CDU a series of velocimetry tests were undertaken with identical PETN powder to that used in the commercial RP-1 detonator, see Fig. 10. In these tests, the particle velocity in the PMMA window was measured for different powder thicknesses for both a very soft-fire and a hard-fire condition. It is has previously been established that a hard-fire from this $5 \mu \mathrm{F} 600 \mathrm{~V} \mathrm{CDU}$ corresponds to an 
asymptotic operation time with respect to further increases of current conduction time [10].

It was observed that with both a soft- and hard-fire, the particle velocity is a ramp wave at $0.5 \mathrm{~mm}$ from the bridgewire with a peak velocity already of approximately twice that of the fastest window velocity created by a powerful bridgeburst in sugar. It is also 17 times that in $0.5 \mathrm{~mm}$ of sugar using the same CDU and bridge. Interestingly, the particle velocity is only slightly lower in the soft-fire case than the hard-fire. By $0.65 \mathrm{~mm}$, more of a shock has formed and in all subsequent cases the hard-fire shock position leads the soft-fire. The right-hand pressure axis in Fig. 10 corresponds to the shock-matched pressure in the PMMA at the particle velocities indicated on the left $[19,20]$. Due to different impedances between unreacted and reacted porous PETN, the shock pressure match to PMMA also varies and this will be discussed later.

The velocity of detonation (VOD) for $0.88 \mathrm{~g} / \mathrm{cc}$ PETN is $5.0 \pm 0.1 \mathrm{~mm} / \mu \mathrm{s}$ [21]. Using the Lawrence Livermore computational thermochemical explosive performance estimation tool CHEETAH, the corresponding steady-state Chapman-Jouguet (CJ) detonation pressure is calculated to be $\approx 6 \mathrm{GPa}$. Using the universal $P-U_{\mathrm{p}}$ curve for reacted explosives products proposed by Cooper [22] allows an approximate shock match calculation to be performed and results in a pressure at the PETN/PMMA-window interface of approximately $6.7 \mathrm{GPa}$. This increase is due to its slightly greater window impedance than the reaction products. For somewhat similar tests with an EBW detonator having a $0.93 \mathrm{~g} / \mathrm{cc}$ PETN fill, it has been previously reported that full detonation was reached after $3 \mathrm{~mm}$ when hard-fired [17]. From these new data in Fig. 10, it appears that steady detonation is achieved in $3.5 \mathrm{~mm}$ when hard-fired, but that in the soft-fire case steady state has not yet quite been achieved by $3.5 \mathrm{~mm}$. However, in both cases a full CJ detonation state has been reached by $6.15 \mathrm{~mm}$.

When steady detonation is occurring, the measured peak pressure in the PMMA is substantially greater than $6.7 \mathrm{GPa}$ and varies between 13-15 GPa. It is presumed that this results from the extended reaction zone in low-density PETN allowing pressure states to be measured that approach, but do not quite reach, the von Neumann (VN) spike [23]. Using the powder shock Hugoniot calculation method suggested by Sheffield et al. [24], the fully compacted unreacted PETN curve of relevance to the VN spike can be estimated. Adding the appropriate gradient Rayleigh line corresponding to the VOD, the Hugoniot intercept corresponding to the VN pressure spike may be found.

Figure 11 shows the resulting curves, CJ points, and VN spike intercepts for both the $0.88 \mathrm{~g} / \mathrm{cc}$ porous material and fully dense PETN for comparison. The VN spike pressure of $13 \mathrm{GPa}$ for the initially low-density powder must also be shock matched into PMMA, and this results in a matched

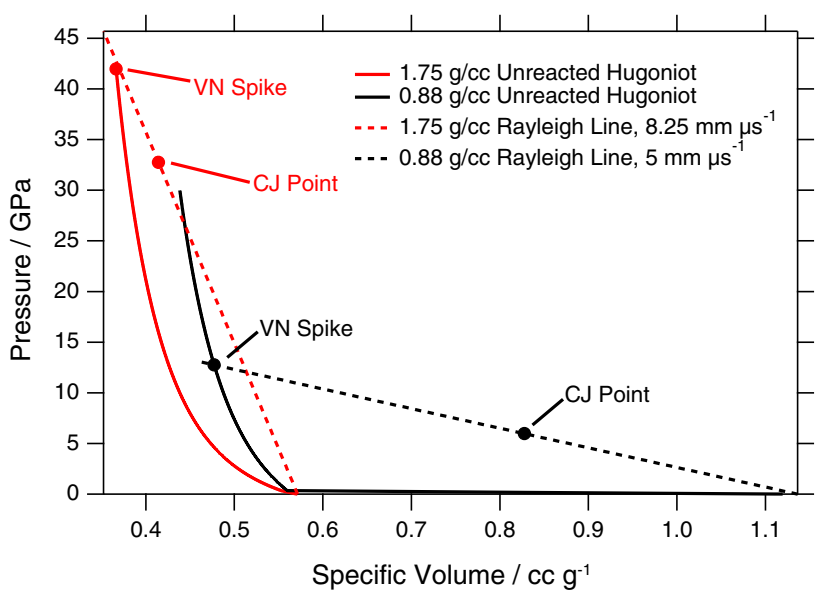

Fig. 11 Illustration of the $\mathrm{VN}$ and CJ points for both $0.88 \mathrm{~g} / \mathrm{cc}$ and fully dense PETN calculated at the corresponding VOD

pressure of $21 \mathrm{GPa}$. It is therefore clear that although states at a pressure considerably greater than the CJ state are resolved, the PDV technique employed is not capable of the temporal resolution required to capture the extremely brief actual VN spike. The extreme drop in particle velocity in nanoseconds that results just behind the spike combined with the temporal averaging that results from the sFFT velocity extraction technique combine to result in a peak extracted velocity that is an underestimate with significant scatter between tests.

Estimates for the reaction zone thickness (the distance from the VN spike lead shock and the sonic locus at the CJ pressure) vary significantly in the literature for PETN of this density from $0.16-0.54 \mathrm{~mm}[25,26]$. At a VOD of $5 \mathrm{~mm} / \mu \mathrm{s}$, this puts the reaction zone duration at between $30-108 \mathrm{~ns}$. The time taken for the pressure to drop from the peak to approximately $6.7 \mathrm{GPa}$ is $83 \mathrm{~ns}$ for the $6.15 \mathrm{~mm}$ curves and therefore lies close to the middle of the estimated reaction zone thicknesses $(0.42 \mathrm{~mm})$.

To examine the velocity of propagation of the growing shock, the position of the wave with respect to the distance from the bridgewire is plotted in Fig. 12 for both the softand hard-fire conditions. For comparison, data from [17] are also plotted for $0.93 \mathrm{~g} / \mathrm{cc}$ PETN in an EBW detonator. It will be seen that in all three cases the velocity is increasing with distance from the bridgewire. In all cases, the soft-fire condition wave lags the equivalent hard-fire one, but this is to be expected since the function time for soft-fired detonators is greater than for hard-fired ones [10]. A fitting form to these data is desired that asymptotes the shock speed $\left(U_{\mathrm{s}}\right)$ to a final steady-state VOD of $5 \mathrm{~mm} / \mu \mathrm{s}$ as a function of time $(t)$ and to assure this the logistic function was used,

$U_{\mathrm{s}}=\frac{\mathrm{VOD}}{1+\mathrm{e}^{-k\left(t-t_{0}\right)}}$.

The position/time points were therefore fit with a leastsquares optimization to the time integral of (1). The cor- 


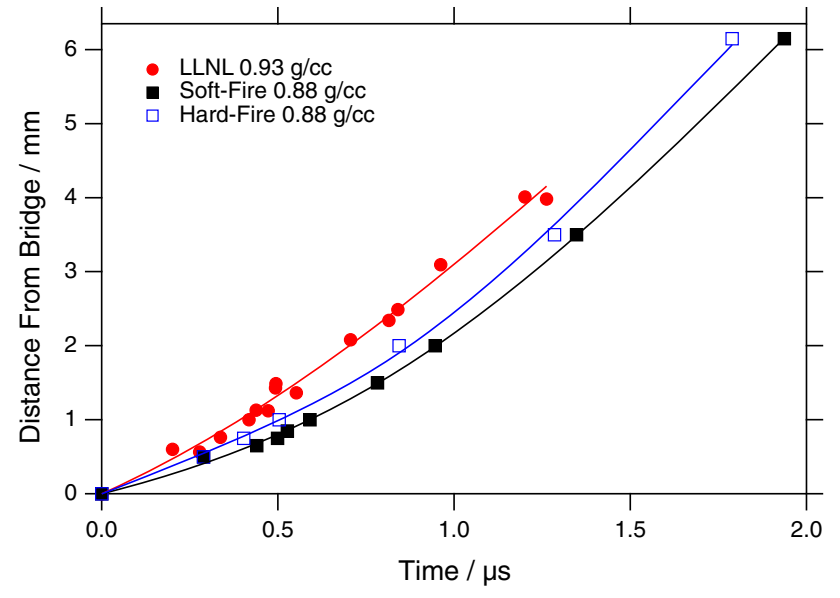

Fig. 12 The shock position versus time points for both the hard- and soft-fire conduction time for $0.88 \mathrm{~g} / \mathrm{cc}$ material compared with data for $0.93 \mathrm{~g} / \mathrm{cc}$ [17]. The least-squares fitting form used is described in the text

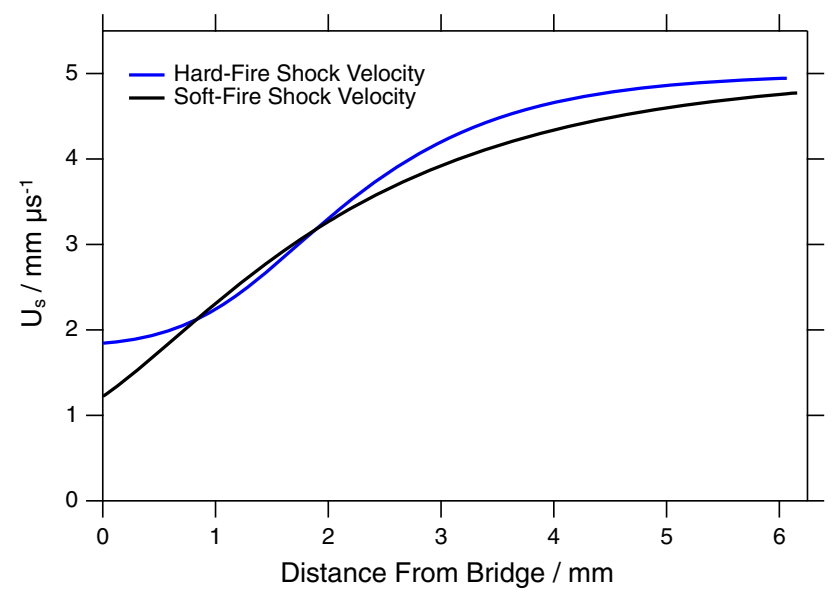

Fig. 13 The corresponding VOD versus distance through the PETN powder bed from the fits presented in Fig. 12. The steady-state (CJ) VOD for $0.88 \mathrm{~g} / \mathrm{cc}$ PETN was defined as $5.0 \mathrm{~mm} / \mu \mathrm{s}$

responding propagation velocities versus distance from the equation (i.e., the derivative of the fit versus the fit) are plotted in Fig. 13. These sparse data are consistent with a shock wave initially traveling at $1.2-1.8 \mathrm{~mm} / \mu \mathrm{s}$ that then accelerates smoothly up toward the expected asymptotic and steady-state velocity of $5 \mathrm{~mm} / \mu$ s through the thickness of the PETN pressing as chemical reactions occur in the explosive behind the lead shock. The rate of growth clearly depends of whether burst was representative of a soft-fire or hard-fire electrical condition.

The reaction buildup process after bridge-burst has been studied using optical means (e.g., photography and streak cameras $[18,27])$ and X-rays [27]. There are marked differences between the findings. The optical observations from outside some form of transparent sleeve or header show a dark time between the bright bridge-burst process and the breakout of a luminous reaction approximately $1 \mathrm{~mm}$ in front of the bridge. This reaction then appears to progress at the speed of full detonation in low-density PETN. Additionally, the optical records suggest a complex time-evolving threedimensional front stemming from a difference in reaction onset between the long axis of the wire and the orthogonal radial direction of the wire. These types of findings have led to the suggestion that there is a dwell time after bridgeburst where a small volume of material in front of the bridge undergoes a bulk thermal explosion resulting from a rapid increase in temperature caused by the exothermic reaction of the PETN [2,28]. Initially this reaction is sufficiently cool that it does not emit significantly at optical wavelengths but there follows a violent thermal explosion which is highly luminous.

To the contrary, the X-ray images show an approximately constant velocity hemispherical density discontinuity wave traveling from the bridge-burst at $\approx 3 \mathrm{~mm} / \mu \mathrm{s}$. For the majority of [27], an RP-80 detonator variant used; however, an RP-1 was used for the comparison between the optical wave traveling at $5 \mathrm{~mm} / \mu$ s that appears to catch the slower density wave derived from X-rays at almost exactly the end of the pressing where the high-density output pellet sits. The new findings presented here, as well as in [17], suggest that the density discontinuity is an initially accelerating reaction wave undergoing a hot-spot-driven shock-to-detonation transition (SDT) process [29-31].

Considering the effects of light propagation and attenuation in low-density PET may partially account for the discrepancy. It has previously been reported that pure PETN powder is highly absorbing at UV and visible wavelengths [32]. It is therefore possible that optical observation on the surfaces of detonators is only sensitive to emissions from relatively close to the surface (except at fine gaps or mechanical joints). To test this, a 1-mm-diameter PMMA fiber was placed at the end of a 6.15-mm RP-1 PETN IP and the bridge hard-fired. As described previously, steady detonation is assured $3.5 \mathrm{~mm}$ from the header giving a $5 \mathrm{~mm} / \mu \mathrm{s}$ detonation distance of at least $2.65 \mathrm{~mm}$. The voltage output from a fast (less than $1 \mathrm{~ns}$ risetime), unamplified $\mathrm{Si}$ (visible) photodiode was digitized together with the other regular electrical signals. The first detectable increase in light signal occurred $360 \mathrm{~ns}$ before the detonation broke out into air. After first being detected, the intensity increased slowly but suddenly became an order of magnitude brighter at the PETN/air interface. Using the VOD, this 360 ns time duration corresponds to a depth of $1.8 \mathrm{~mm}$ back into PETN at a density of $0.88 \mathrm{~g} / \mathrm{cc}$ from which an event as bright as a full detonation is first observable with a regular unamplified photodiode.

It is difficult to compare an optical camera's detectable dynamic range with a photodiode/digitizer combination, but it is conceivable that if the camera settings were configured to avoid gross saturation upon detonation breakout that the light from relatively low overall intensity events, such as an initial 
ignition event or a weak but building chemical reaction, may fail to register through a maximally thick distance of powder. In this case, only the light from reactions closer to the powder extents that have built in strength as they travel, and hence are fairly close to detonation conditions, would be recorded. Due to interactions between corner turning phenomena, camera sensitivity, and the wavelength-dependent absorption of PETN, it is possible that a complex optical breakout pattern could occur close to the header compared with a relatively simple density resolved one. Given the greater hypotenuse reaction path length to the detonator sleeve than just along the axis, the flattening behavior of reaction waves, and the rapid rate of detonation buildup in the final stages, it is also reasonable that the apparent phase velocity of the reaction front along the sleeve would differ from measurements purely along the detonator axis.

The functioning of special SE-1 detonators (a design almost identical to the RP-1) was investigated in [33] as a function of PETN particle size. The key diagnostic was timeof-arrival optical fibers placed either in the header (radially separated from the bridge), in the sleeve wall (both radially and axially separated from the bridge), or in plastic plugs placed on top of variable height PETN pressings (axially separated). In all cases, a constant porous density of $0.9 \mathrm{~g} / \mathrm{cc}$ PETN was maintained.

Firstly, it was discovered that the fibers in the header and sleeve exhibited difficult to interpret time-of-arrival signals due to the corner turning behavior of the detonation. The corner turning phenomenon is a complex interaction of a divergent explosive reaction wave and the local and changing velocity of propagation caused by the pressure release processes operating in the finite reaction zone length. That is, owing to the difference between the volume and surface area of a burning region with fixed chemical energy per unit mass, small dimension expanding regions propagate more slowly than larger ones. Additionally, the finite reaction zone between the lead shock and the sonic locus (the sound-speed boundary where additional energy from reactions can catch up to and support the shock) allows pressure release processes from the edges to cause reaction front curvature and slow the entire detonation. Indeed, this is the definition of an non-ideal explosive.

The result of these processes is a complex slowing of the speed of propagation in areas of high curvature, such as those close to an exploding bridgewire. It is believed that this accounts for the difficult to interpret optical signals results in both $[27,33]$. In both cases, the optical observation was made at a sufficient distance from the ignition site that either a very strong but still building reaction shock or a full detonation arrived, but the resulting spatial time of arrival at the walls was complex. In the case of the purely axial time-of-arrival measurements presented in [33], similar times of arrival data were obtained to the new results presented in this paper.
The second very significant finding was that after an ignition process, the duration of the acceleration phase in the building reaction was a very strong function of the PETN surface area. In fact, PETN with a surface area (SA) of $10,000 \mathrm{~cm}^{2} \mathrm{~g}^{-1}$ transitioned to a steady-state detonation in a time too fast for the experimental arrangement to measure, while PETN with a SA of $3000 \mathrm{~cm}^{2} \mathrm{~g}^{-1}$ needed $\approx 5 \mathrm{~mm}$ of run length.

For comparison, the PETN used in this study had a SA of $5800 \mathrm{~cm}^{2} \mathrm{~g}^{-1}$ while the PETN used in the RP- 80 (relevant to much of [27]) is lower at $\approx 3000 \mathrm{~cm}^{2} \mathrm{~g}^{-1}$. Comparing the RP-1 PETN with the data in [33] suggests a run distance to detonation of $3.5 \mathrm{~mm}$ is reasonable for the RP-1 PETN. Given the $4.98 \mathrm{~mm}$ long low-density pressing in the RP-80 and the lower SA, it is possible that the reaction has barely transitioned to a steady state by the time the shock reaches the high-density output pellet. Additionally, such a lower pressure lead shock may result in a short run-to-detonation being required in the high-density $\mathrm{OP}$ (with associated increased jitter in growth time) rather than the desired prompt CJ detonation at the interface. Indeed, consulting [27], such a brief hesitation at the pellet interface is observed. A lack of steady detonation in the IP would therefore explain both the longer excess transit time (ETT) for the RP-80 (1160 ns) compared with the RP-1 (750 ns) and the increased standard deviation in measured total function time (125 ns for the RP- 80 and $25 \mathrm{~ns}$ for the RP-1).

The PDV tests on the longer pressings allowed the total function time of just a $6.15 \mathrm{~mm}$ PETN IP to be measured (bridge-burst to axial detonation breakout into air), and the result was $1792 \pm 40 \mathrm{~ns}$. Since the initial shock velocity from bridge-burst to $3.5 \mathrm{~mm}$ grew from $1-4.5 \mathrm{~mm} / \mu \mathrm{s}$, an average velocity of $\approx 2.75 \mathrm{~mm} / \mu \mathrm{s}$ is assumed. This results in a total time of $1273 \mathrm{~ns}$ until steady-state detonation is achieved. The time taken for the same distance at full detonation velocity is $700 \mathrm{~ns}-\mathrm{a} 573 \mathrm{~ns}$ discrepancy. The time to detonate $6.15 \mathrm{~mm}$ at a VOD of $5 \mathrm{~mm} / \mu \mathrm{s}$ is $1230 \mathrm{~ns}$. This simple bi-linear approximation results in a $11 \mathrm{~ns}$ discrepancy between the measured function time (1792 ns) and the calculated time for steadystate detonation (1230 ns) plus the build up time (573 ns).

Since the measured total ETT for this hard-fired RP-1 at $600 \mathrm{~V}$ is $854 \mathrm{~ns}$, this leaves $\approx 281 \mathrm{~ns}$ still unaccounted for and it must occur in the OP region. Shock sensitivity data (Pop-plot) for PBX 9407 (the output explosive in an $\mathrm{RP}-1)$ at a density of $1.6 \mathrm{~g} / \mathrm{cc}$ are sparse and relatively noisy, particularly at small runs-to-detonation [34]. Shock matching a 6 GPa PETN detonation into PBX 9407 results in a pressure of 7.5 GPa. The Pop-plot data do not extend to so great a pressure, but extrapolation suggests that detonation would occur at only $0.3 \mathrm{~mm}$ (less than $100 \mathrm{~ns}$ ).

However, it must be recalled that Pop-plot data are for a fully supported constant shock pressure input. The shock velocity profiles shown in Fig. 10 show classic Taylor wave 
responses behind the lead shock and so are clearly not a fully supported shock input into the OP. Such high pressure, but short-shock, inputs into explosives lead to an extended run-to-detonation distance or even failure to detonate. Some validation of a relatively long buildup to detonation between low-density PETN and a high-density OP is found in [27] where the velocity of propagation measured by radiography appears to show a stutter in the density wave at the transition of the IP and OP of $\approx 100 \pm 50 \mathrm{~ns}$.

The SA-dependent acceleration in the reaction shock accounts for the some of the ETT in PETN-based EBW detonators and is also broadly supported by the findings of and data presented in [26]. However, in that paper some of the ETT is attributed to a detonation occurring relatively promptly after the bridge-burst, with the argument that initially the velocity of detonation is slower than under CJ conditions due to detonation curvature and corner turning effects. The new data presented here and in [17] do not have sufficient resolution to fully prove or disprove this explanation, but do indicate that the transition to steady-state detonation occurs relatively far from the bridge where the curvature effects are less significant. However, the data in [33] do demonstrate that in some high SA PETN steady-state detonation can occur promptly and without a detonation curvature delay effect along the axis of the detonator (i.e., with imperceptibly low ETT).

Unfortunately, from the point of view of an intuitive understanding of the particle surface area to void shape, the particle morphology of the PETN used in detonators is not even remotely spherical. Instead, typical morphologies are everything from rhomboids to needle-like crystals with high aspect ratios $[35,36]$. This makes estimation of surface area per unit mass highly dependent on the method used. For example, reference [33] used sample gas permeability. More recently, a variety of inert gas absorption techniques have been used since these provide a better metric of the true SA; however, since there are a number of different methods used and they do not result in the same answer, comparisons between different papers are still challenging [35].

Given that it has been reported since the early 1960s that improved performance of EBW detonators could be obtained by using higher SA PETN [18,33,37], why do all designs not currently use such high SA PETN? A partial answer is that although the buildup to detonation happens more promptly in high SA PETN, the burst power (and energy) to function the detonator also significantly increases [33] and this creates practical implementation difficulties with respect to the CDU and electrical components as well as the electrical insulation required. Therefore, the choice of the PETN for a given detonator design is a compromise between adequate timing (ETT standard deviation) and volume/mass/cost CDU considerations.
Significantly, it has been discovered that the shock sensitivity of PETN is almost independent of particle morphology for a fixed SA [38]. Less surprisingly, from a constant energy density point of view, it has been found that the final VOD in PETN powder is almost independent of SA and is purely a function of pressing density [33].

Howe et al. have previously published the observation that an increased shock pressure is required to ignite fine explosive particles compared to coarse, but that burning in fine particles accelerates more quickly [39]. The discussion in that paper regarding reaction buildup in explosives assumes numerous discrete energy localization sites (hot-spots) that drive rapid chemistry. This key paper shows that the buildup rate of reaction correlates well with the surface area of the explosive particles, not with the hot-spot density or hot-spot sizes per se (although they are often related). It also addresses the issue of why coarse particles are more sensitive to shocks than fine. Converting the explanation into terms relevant to the research presented here, their observation was that for a fixed density of powder, voids between smaller particles were smaller and vice versa. Thus, as a shock of a fixed pressure compacts the material, the distance required to crush the void is also smaller for smaller particles. A fixed pressure onedimensional shock by definition exerts a constant force per unit area and so the work done on the void is a constant force times a distance that depends on the void size. Since that distance is shorter for smaller voids (higher SA powder), the deposited energy is smaller and so the temperature rise must also be lower. Hence, smaller voids (from smaller particles), although more numerous, are less efficient at starting initial reaction. However, any growing nascent reaction that does occur immediately accesses a relatively larger surface area of as yet unreacted material and this accelerates the growth more quickly than in similar powder densities with larger voids.

A consideration of Frank-Kamenetski thermal explosion theory also predicts that smaller hot-spots are less likely to initially propagate than larger ones of the same temperature due to the volume versus surface area argument mentioned earlier [40]. Finally, if the density of a powder is held constant, coarse particle beds contain fewer, but larger voids than smaller particles with smaller but more numerous voids. A shock of fixed pressure results in a fixed total amount of energy being deposited in the shocked material. In the limit, therefore, a fine enough powder will begin to resemble a homogeneous solid where the localization sites are so numerous that energy ceases to be localized at all and is distributed equally throughout the mass. This results in a minimal temperature rise to drive reaction chemistry.

Although it has been shown that some of the ETT can be accounted for by the SDT buildup process (which presumably must be highly reproducible for a given particle SA and pressing density), there is still the matter of the ignition 


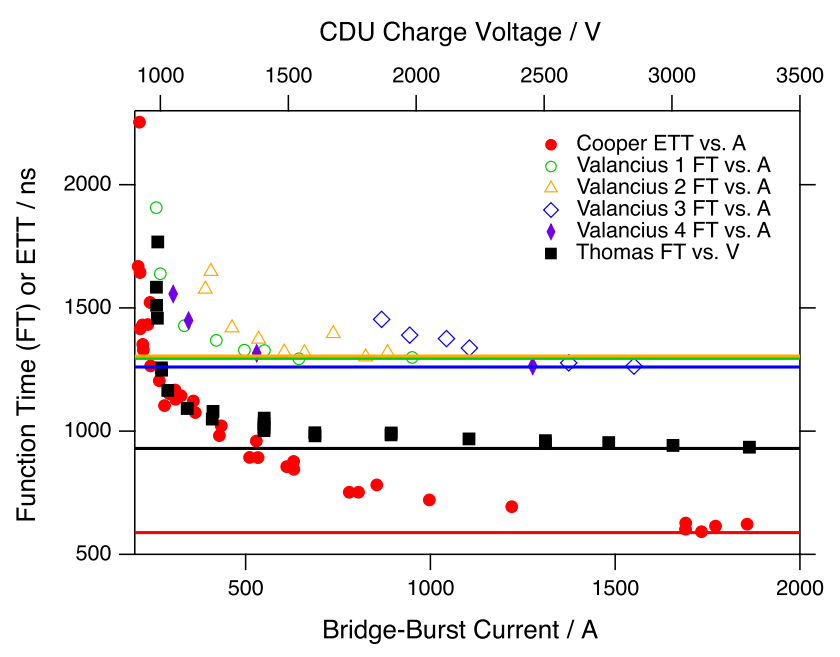

Fig. 14 The ETT or total function time for a variety of EBW detonators versus bridge-burst current or CDU charge voltage. Data from [42-44]

process produced by the bridge-burst. It has been observed previously that the functioning of EBW detonators is not just a traditional SDT process [2]. The results and discussion presented in this paper require significant clarification of this statement since the buildup to detonation is clearly a traditional hot-spot energy localization SDT process.

What is not traditional is the ignition process that must occur between the bridge-bursting and the onset of SDT. Specifically, it was already known [41] and has been demonstrated again in Sect. 3.1 of this paper that the shock pressure from a bursting wire is a function of the energy at burst (practically speaking CDUs with more stored energy result in stronger shock outputs into the powder). However, the total function time of a hard-fired EBW detonator is often reported as being constant for a fixed design essentially irrespective of how large an energy CDU is used. There is no common shock ignition process in polycrystalline explosives where a stronger and stronger initial shock does not result in a faster and faster buildup to detonation.

It is therefore clear that either some rate-limiting step occurs in EBW detonators where, once initial ignition occurs, no practical extra amount of energy delivered by the action of a bursting fine wire can accelerate the process faster. Or, more radically, that the function time of a hard-fire is a sufficiently weak function of CDU energy that it approaches the standard deviation in regular function time and so is largely hidden. This second explanation would require a revised, and less absolute, statement regarding hard-fire ETT in EBWs: that no practical increase in CDU energy above that required for hard-fire results in a significant change in total function time.

Figure 14 reveals more about this important hard-fire function time issue. In it, data from three sources are cross-plotted to reveal the change in function time or ETT for a vari-

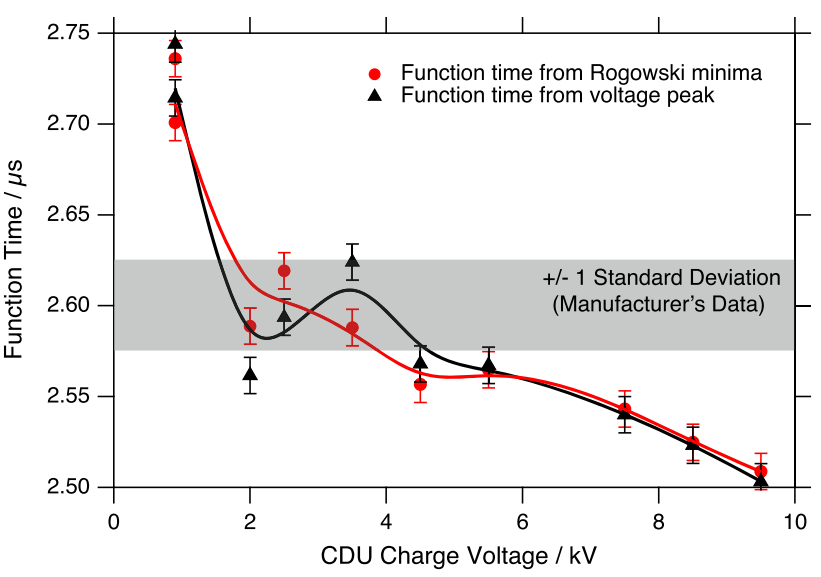

Fig. 15 RP-1 total total function time versus CDU charge voltage. The smoothing spline lines through the data are just a guide for the eye

ety of EBWs as a function of either burst current or CDU charge voltage. To a first order, burst current and CDU voltage are comparable because, for a large CDU, the initial rate of current rise is approximately linear with charge voltage. Significantly, in about half the curves it appears that the function time is still slowly decreasing as the voltage or current is increased, while in the other half it appears that constant value has been reached. Unfortunately, error bar magnitude data are not given in the references and so this statement cannot be quantified.

Part of the trouble with measuring this phenomenon is that most CDUs in day-to-day use have a fairly small working voltage range (often as a result of the triggered gas switch used) and so data are simply not available outside of a fairly narrow range of burst conditions. For example, many common CDUs are rated between about $1.5-3 \mathrm{kV}$, representing only a factor of four change in stored energy.

In an effort to expand the measurement range, two CDUs with more modern solid state, and wider working range, S-38 optically triggered switches were used. The first was the CDU used for the sugar tests above $(0.9-3.5 \mathrm{kV})$ while the second was a $2 \mu \mathrm{FCDU}$ with a charging range between $2-10 \mathrm{kV}[3]$. RP-1 detonators were test fired under a wide variety of charge conditions and the total function time assessed from the time difference between bridge-burst voltage signal and the arrival at the output face of the detonation using a PVDF sensor adhered to the output pellet with low-viscosity glue [10]. The results using two methods of calculating the total function time (the Rogowski sensor minima at burst and the peak voltage spike) are shown in Fig. 15. Here the error bars represent the uncertainty in the measurement. Additionally, the standard deviation in total function time for this detonator is under normal firing conditions $\approx 25 \mathrm{~ns}$ and is represented by the gray shaded area in the plot.

It is apparent that an increased energy at burst does have a small but measurable effect on overall ETT. From the stud- 
ies of bursts in inert sugar, two things contribute to this relatively minor effect (a 250 ns change in total detonator function time or $\approx 10 \%$ ). It has been demonstrated that even the most powerful bursts in inert powders result in ramped shocked powder compaction speeds of $600-1000 \mathrm{~m} / \mathrm{s} \mathrm{com-}$ pared with $>1000 \mathrm{~m} / \mathrm{s}$ within the first half millimeter of powdered PETN. Further, from Table 1, it is clear that the inert compaction speed is only a relatively weak function of CDU voltage. This implies that any building reaction in the PETN quickly accelerates away from the bridge-burstinduced compaction site and so obtains little practical energy support from behind the reaction front (i.e., the PETN reaction efficiency, and hence acceleration of the reaction front, is much greater than the bridge-burst effect into porous powder). Secondly, and related to the previous observation, the maximum particle velocity in a PMMA window $0.5 \mathrm{~mm}$ from the bridge-burst in inert powder was only $270 \mathrm{~m} / \mathrm{s}$ compared with almost $500 \mathrm{~m} / \mathrm{s}$ from reacting PETN at the same distance. This again implies that the energy released by PETN ignition and growth of reaction processes are more effective at producing accelerating reaction growth than the processes available from the action of an exploding wire.

This important new observation for the first time explains why EBW detonators are useful in practical situations. The action of electrically exploding a fine wire is highly efficient at starting a violent reaction in powdered explosives such as PETN, but the nature of the response of low-density powders to shocks generated from such exploding wires limits the duration where they are actually relevant to accelerating the building reaction in the explosive. Thus, over a relatively wide range of firing conditions (wire burst conditions) the acceleration of the SDT process in highly porous PETN (or other similar explosives) is largely unaffected leading to an asymptotic and highly reproducible total function time for the detonator.

It is therefore important to reiterate that only the extreme range of burst conditions imposed revealed this reduction in ETT and that even then the total change at extreme charge voltage was only about four times the standard deviation measured for the detonator. Future studies will focus specifically on the effect the porous IP detonation process has on the important SDT process in the solid output pellet as well as obtaining better understanding of the ignition processes occurring immediately surrounding the bridgewire.

\section{Conclusions}

It has been shown that the electrical explosion of thin gold wires by the application of high currents and voltages from a capacitor discharge unit generates strong ramp waves in thin low-density sugar pressings. Powerful bridge-bursts into powdered sugar with a density of $0.88 \mathrm{~g} / \mathrm{cc}$ result in ramp wave particle velocities of $70-270 \mathrm{~m} / \mathrm{s}$ in an adjacent PMMA window that would correspond to window pressures of $\approx 0.25-1 \mathrm{GPa}$ if the wave was instead a shock. A comparable bridge-burst and a pure electrical arc resulted in an arc-produced particle velocity only $25 \%$ lower than the bridge-burst at $0.85 \mathrm{~mm}$ from the bridge. However, the peak electrical power was much smaller in the case of the arc discharge since there was no burst event.

The effects of cable inductance and ring-up time (i.e., a longer firing cable) on the output of the bridge-burst into inert powder were found to be dramatic, even though a burst still resulted that would be adequate to hard-fire an EBW detonator. Very energetic bursts resulted in little particle velocity attenuation out to $1 \mathrm{~mm}$, while the velocities did drop significantly between 0.5 and $1 \mathrm{~mm}$ for more marginal bursts. The shock compaction velocity in the inert sugar beds from moderate to very strong bursts was found to be relatively constant at $600-1000 \mathrm{~m} / \mathrm{s}$, but this was not the case for marginal bursts where a slower and quickly reducing velocity was measured (400-280 m/s).

Particle velocities in the PMMA windows of as little as $35 \mathrm{~m} / \mathrm{s}$ were measured at powder thicknesses of 0.5 and $0.85 \mathrm{~mm}$ for bursts that would still have resulted in a hard-fired EBW detonator. Bursts corresponding to threshold conditions had window particle velocities as low as $7 \mathrm{~m} / \mathrm{s}$ at $0.85 \mathrm{~mm}$. Conditions for just below threshold bursts also resulted in very similar particle velocity profiles to those for threshold bursts.

The key finding in a real explosive powder (PETN) is that after a distance of $0.65 \mathrm{~mm}$ from the bridge a clear hot-spotdriven SDT was observed. This transition phase accounts for approximately $66 \%$ of the excess transit time that was somewhat unaccounted for previously. For the powder particle SA used, the shock transitioned to a detonation after approximately $3.5 \mathrm{~mm}$. A review of the literature suggests that this rate of SDT buildup is affected by the SA of the powder and that with high enough SA a SDT can occur almost instantly (with a correspondingly low ETT), but at the expense of requiring a more powerful bridge-burst to start the ignition process. It is proposed, but not conclusively proven, that the remaining ETT ( $34 \%$ or $\approx 280 \mathrm{~ns}$ ) in the OP is due to a long buildup to detonation in the relatively insensitive die-pressed PBX 9407 used in the RP-1. This aspect will be addressed more fully in future studies of EBW detonators.

This paper questions the accepted rule-of-thumb that the function time of a hard-fired EBW detonator is unchanging with increased bridge-burst energy above a critical value. Instead it is demonstrated that a small, but measurable, reduction in ETT occurs with increasingly energetic bridge-bursts and that this small effect results from the inherent response of a low-density high-specific surface area explosive powder coupled with the relatively small variance in burst conditions produced by common CDUs. This is significant because it 
brings the behavior of EBW detonators fully into accordance with firmly established behaviors and reaction mechanisms observed in other explosive devices and systems. That is, a greater energy stimulus input into the explosive does result in a faster transition to detonation, but in the specific case of EBW detonators, the nature of the low-density powder used limits the effectiveness of this increased energy deposition in changing the resulting detonator function time.

In summary, consideration of new evidence recently available, presented both in this paper and other recent papers by others, for the first time allows for the processes occurring after ignition and thermal explosion in PETN from the action of an exploding bridgewire to be explained using established phenomena commonly observed in other explosive systems.

Acknowledgements The authors wish to thank Eva Baca for undertaking the sugar pressing, Micah Jakulewicz for performing some of the tests, and Eric Heatwole for advice on the VOD data analysis.

Funding Research presented in this article was supported by the Laboratory Directed Research and Development program of Los Alamos National Laboratory under Project No. 20210189ER.

\section{Declarations}

Conflict of interest The authors declare that they have no conflict of interest.

Open Access This article is licensed under a Creative Commons Attribution 4.0 International License, which permits use, sharing, adaptation, distribution and reproduction in any medium or format, as long as you give appropriate credit to the original author(s) and the source, provide a link to the Creative Commons licence, and indicate if changes were made. The images or other third party material in this article are included in the article's Creative Commons licence, unless indicated otherwise in a credit line to the material. If material is not included in the article's Creative Commons licence and your intended use is not permitted by statutory regulation or exceeds the permitted use, you will need to obtain permission directly from the copyright holder. To view a copy of this licence, visit http://creativecomm ons.org/licenses/by/4.0/.

\section{References}

1. Varosh, R.: Electric detonators: EBW and EFI. Propellants Explos. Pyrotech. 21, 150-154 (1996). https://doi.org/10.1002/prep. 19960210308

2. Rae, P.J., Dickson, P.M.: A review of the mechanism by which exploding bridge-wire detonators function. Proc. R. Soc. A 475, 20190120 (2019). https://doi.org/10.1098/rspa.2019.0120

3. Rae, P.J., Rettinger, R.C.: The effects of air gaps and inert layers on exploding bridgewire detonator function. J. Energ. Mater. (2020). https://doi.org/10.1080/07370652.2020.1825545

4. Tucker, T.J.: Spark initiation requirements of a secondary explosive. Ann. N. Y. Acad. Sci. 152(1), 643-653 (1968)

5. Tucker, T.J., Kennedy, J.E., Allensworth, D.L.: Secondary explosive spark detonators. 7th Symposium on Explosives and Pyrotechnics, Philadelphia, PA, USA (1971)
6. Lee, E., Drake, R.: Relationship between exploding bridgewire and spark initiation of low density PETN. AIP Conf. Proc. 1793, 040012 (2017). https://doi.org/10.1063/1.4971506

7. Leopold, H.S.: Initiation of explosives by exploding wires. V. Effect of wire material on the initiation of PETN by exploding wires. NOLTR 64-146, AD609449, U. S. Naval Ordnance Laboratory White Oak, Maryland (1964)

8. Leopold, H.S.: Initiation of explosives by exploding wires. VI. Further effects of wire material on the initiation of PETN by exploding wires. NOLTR 65-1, AD463360, U. S. Naval Ordnance Laboratory White Oak, Maryland (1965)

9. Webb, F.H., Hilton, H.H., Levine, P.H., Tollestrup, A.V.: The electrical and optical properties of rapidly exploded wires. In: Exploding Wires: Volume 2, pp. 37-76. Boston, USA (1962)

10. Rae, P.J., Feagin, T.A., Heatwole, E.M.: Investigating the minimum post-burst energy required to function an exploding bridgewire detonator. J. Appl. Phys. 128, 033301 (2020). https://doi.org/10.1063/ 5.0006804

11. Strand, O.T., Goosman, G.R., Martinez, M.E., Whitworth, T.L.: Compact system for high-speed velocimetry using heterodyne techniques. Rev. Sci. Instrum. 77, 083108 (2006). https://doi.org/10. $1063 / 1.2336749$

12. Dolan, D.H.: Extreme measurements with photonic Doppler velocimetry (PDV). Rev. Sci. Instrum. 91, 4363 (2020). https:// doi.org/10.1063/5.0004363

13. Rae, P.J.: The action-integral and energy to explode short gold wires in ambient air. Phys. Plasmas 28, 033509 (2021). https://doi.org/ 10.1063/5.0041067

14. Dolan, D.H.: Accuracy and precision in photonic Doppler velocimetry. Rev. Sci. Instrum. 81, 053905 (2010). https://doi.org/ 10.1063/1.3429257

15. Chapman, D.J., Eakins, D.E., Williamson, D.M., Proud, W.: Index of refraction measurements and window corrections for PMMA under shock compression. AIP Conf. Proc. 1426, 442 (2012). https://doi.org/10.1063/1.3686313

16. Kennedy, J.E., Thomas, K.A., Early, J.W., Garcia, I.A., Lester, C., Burnside, N.J.: Mechanisms of exploding bridgewire and direct laser initiation of low density PETN. In: Proceedings of the 29th International Pyrotechnics Seminar, pp. 781-785 (2002)

17. Roeske, F., Benterou, J., Lee, R., Roos, E.: Transition to detonation in exploding bridgewire detonators. Tech. Rep. UCRL-JC-151285, LLNL (2003)

18. Blackburn, J.H., Reithel, R.J.: Exploding wire detonators: sweeping-image photographs of the exploding bridgewire initiation of PETN. In: Exploding Wires: Volume 3, pp. 153-174. Boston, USA (1964)

19. Barker, L.M., Hollenbach, R.E.: Shock-wave studies of PMMA, fused silica, and sapphire. J. Appl. Phys. 41, 4208-4226 (1970). https://doi.org/10.1063/1.1658439

20. Marsh, S.P.: LASL Shock Hugioniot Data. University of California Press, Berkeley (1980)

21. Hornig, H.C., Lee, E.L., Finger, M., Kurrle, J.E.: Equation of state of detonation products. In: Short, J.M., Ohm, W.J. (eds.) Fifth Sympoium (International) on Detonation, pp. 503-512. Pasadena, CA, USA (1970)

22. Cooper, P.W.: Explosives Engineering. Wiley, New York (1996)

23. Forbes, J.W.: Shock Wave Compression of Condensed Matter: A Primer. Springer, Berlin (2012)

24. Sheffield, S.A., Gustavsen, R.L., Anderson, M.U.: Shock loading of porous high explosives. In: Davidson, L., Horie, Y., Shahinpoor, M. (eds.) High-Pressure Shock Compression of Solids IV. Springer, USA (1997)

25. Dinegar, R.H.: Detonation velocity of PETN in small confined cylindrical charges. Propellants Explos. 1, 97-100 (1976). https:// doi.org/10.1002/prep.19760010504 
26. Drake, R.C.: Assessing the effect of the role of detonation wave curvature on the firing times of high voltage detonators. AIP Conf. Proc. 1979, 100010 (2018). https://doi.org/10.1063/1.5044882

27. Smilowitz, L., Henson, B.F., Remelius, D., Bowlan, P., Suvorova, N., Allison, J., Cardon, D., Freeman, M., Mariam, F., Meijer, W., Morris, C., Neukirch, L., Prestridge, K., Sandstrom, M., Saunders, A., Schurman, T., Tainter, A., Tang, Z., Trouw, F., Tupa, D., Tybo, J., Uliano, R.: Experimental observations of exploding bridgewire detonator function. J. Appl. Phys. 128, 215901 (2020). https://doi. org/10.1063/5.0021288

28. Smilowitz, L., Remelius, D., Suvorova, N., Bowlan, P., Oschwald, D., Henson, B.F.: Finding the lost-time in detonator function. Appl. Phys. Lett. 114, 104102 (2019). https://doi.org/10.1063/1.5088606

29. Campbell, A.W., Davis, W.C., Ramsey, J.B., Travis, J.R.: Shock initiation of solid explosives. Phys. Fluids 4, 511-521 (1961). https:// doi.org/10.1063/1.1706354

30. Belmas, R., Plotard, J.P.: Physical origin of hot spots in pressed explosive compositions. J. Phys. IV Proceedings, EDP Sciences, 05(C4), pp. C4-61-C4-87 (1995)

31. Zukas, J.A., Walters, W.P.: Explosive Effects and Applications, 1st edn. Springer, New York (1997)

32. Feagin, T.A., Rae, P.J.: Optical absorption in polycrystalline PETN, RDX, HMX, CL-20 and HNS and its possible effect on exploding bridgewire detonator function. J. Energ. Mater. 38(4), 395-405 (2020). https://doi.org/10.1080/07370652.2020.1716110

33. Mohler, J.H., Moodie, W.J., Haws, L.D., Hall, G.F.: Characterization of high explosives by observing growth to detonation. Combust. Flame 32, 285-294 (1978). https://doi.org/10.1016/ 0010-2180(78)90103-7

34. Gibbs, T.R., Popolato, A.: LASL Explosive Property Data. University of California Press, Berkeley (1980)

35. Young, S.: Method development and validation for measuring the particle size distribution of pentaerythritol tetranitrate (PETN) powders. Tech. Rep. SAND2005-6487, Sandia National Laboratory (2006)

36. Brown, G.W., Sandstrom, M.M., Giambra, A.M., Archuleta, J.G., Munroe, D.C.: Thermal analysis of pentaerythritol tetranitrate and development of a powder aging model. Tech. Rep., Los Alamos National Laboratory, NM, USA, LA-UR-09-05019 (2009)
37. Tucker, T.J.: Exploding wire detonators: threshold burst current dependence upon detonator and environmental parameters. In: Exploding Wires: Volume 4, pp. 211-232. Boston, USA (1967)

38. Dinegar, R.H., Rochester, R.H., Millican, M.S.: Effect of specific surface on the shock sensitivity of pressed granular PETN. Explosivstoffe 9, 188 (1963)

39. Howe, P., Frey, R., Taylor, B., Boyle, V.: Shock initiation and the critical energy concept. In: Edwards, D.J., Jacobs, S.J., (eds.) Proceedings of the 6th International Detonation Symposium, pp. 11-19. Coronado, CA, USA (1976)

40. Frank-Kamenetski, D.A.: Calculation of thermal explosion limits. Acta Phys.-Chim USSR 10, 365 (1939)

41. Wilkins, P.R., Frank, A.M., Lee, R.S., May, C.: Dynamic shock front measurements and electrical modeling of the exploding gold bridge wire in a detonator. Tech. Rep. UCRL-JC-151976, LLNL (2003)

42. Cooper, P.W., Owenby, R.N., Stofleth, J.H.: Excess transit time as a function of burst current in an exploding bridgewire detonator. In: Proc. of Fourteenth Symposium on Explosives and Pyrotechnics, pp. 3.1-3.10. San Francisco, CA, USA (1990)

43. Thomas, K.A., Liechty, G.H., Jaramillo, D.C., Munger, A.C., McHugh, D.C., Kennedy, J.E.: On the use of an ER-213 detonator to establish a baseline for the ER-486. Tech. Rep. LA-UR-14-26570, LANL (2014)

44. Valancius, C.J., Garasi, C.J., OMalley, P.D.: Power and energy of exploding wires. AIP Conf. Proc. 1793, 040040 (2017). https://doi. org/10.1063/1.4971534

Publisher's Note Springer Nature remains neutral with regard to jurisdictional claims in published maps and institutional affiliations. 